\title{
Self-Destructing "Mothership" Capsules for Timed Release of Encapsulated Contents
}

\author{
Matthew B. Dowling, ${ }^{\dagger, \S}$ Anand S. Bagal, ${ }^{\ddagger} \S$ and Srinivasa R. Raghavan $*, \dagger$ \\ ${ }^{\dagger}$ Fischell Department of Bioengineering and Department of Chemical and Biomolecular Engineering, University of Maryland, \\ College Park, Maryland 20742-2111, United States
}

ABSTRACT: We describe a new class of hierarchical containers that are formed via single-step assembly and, at a later time, self-destruct because of their packaged contents. These containers are spherical capsules formed by electrostatic complexation of the anionic biopolymer, gellan gum, with the cationic biopolymer, chitosan. The capsules are termed "motherships" and are engineered to carry a cargo of much smaller containers (e.g., nanoscale liposomes ("babyships")), within their lumen. Additionally, we package an enzyme, chitosanase, in the capsule that is capable of degrading polymeric chitosan into short oligomers. Thereby, we create

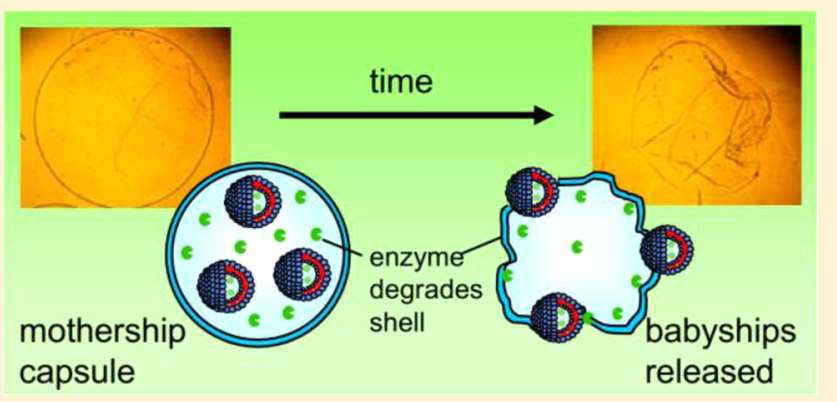
motherships that self-destruct, liberating their cargo of babyships into the external solution. The time scale for self-destruction can be engineered based on the internal concentration of enzyme. The motherships are stable when stored in a freeze-dried form and can be readily dispersed into water or buffer solutions at a later time, whereupon their "internal clock" for self-destruction is initiated. The above concept could be useful for the triggered release of a variety of payloads including drugs, biological therapeutics, cosmetics, and flavor ingredients.

\section{INTRODUCTION}

Polymeric capsules consisting of a liquid core surrounded by a solid polymeric shell are being increasingly studied by researchers. ${ }^{1,2}$ Payloads such as drugs, therapeutics, cosmetics, and flavor ingredients can be packaged in the liquid core of these capsules and delivered in a sustained manner across the outer capsule shell to the external medium. Such capsules are finding applications in numerous industries, including pharmaceuticals, nutraceuticals, agrochemicals, and consumer products. Capsules with new architectures are also being actively explored. One such architecture is to have large polymer capsules that encapsulate smaller container structures. For example, vesicles or liposomes are typically nanoscale containers enclosed by a lipid bilayer, and these can be encapsulated within microscale polymer capsules. ${ }^{3-6}$ There are many advantages to this hierarchical design: for example, in the context of biomedical applications, the capsule shell may protect the liposomal cargo from enzymes or immune cells. At the same time, liposomes may extend the functionality of capsules (e.g., because they are more effective at encapsulating hydrophobic anticancer drugs than the capsules themselves). Finally, the combination of transport resistances from the lipid bilayer and the capsule shell can prolong the release of drug loaded in the liposomes.

A further challenge is to engineer polymer capsules with shells that degrade under the effect of stimuli such as light, temperature, $\mathrm{pH}$, and chemical cues. ${ }^{1,2,6-9}$ Such responsive capsules will hold onto their payload until a stimulus (typically external) is applied and the entire payload is released en masse. A different but related concept is to design self-immolative or self-destructing systems that are immune to external effects but break down because of an internal trigger. A few examples of self-destructing capsules have been published. In the design of De Geest et al., ${ }^{10,11}$ capsules were created by coating a dextranbased microgel with a polymer membrane using layer-by-layer (LbL) deposition. The microgel degraded on hydration, which increased the osmotic pressure and thereby induced the rupture of the capsule. Self-degrading capsules were also designed by Borodina et al. by incorporating enzyme-loaded calcium carbonate particles within the lumen of polymer microcapsules prepared by the LbL technique. ${ }^{12}$ A calcium-chelating agent was then used to dissolve the particles and release the enzymes, which in turn digested the capsule shell and ruptured the capsules. The above schemes for creating self-destructing capsules are elegant but complex. For example, LbL requires a series of labor-intensive incubation and washing steps to form the capsule shell. The use of multistep or aggressive conditions for capsule preparation can also be problematic if the payload to be delivered consists of sensitive biomolecules or drugs.

In this article, we demonstrate a simple, mild process to generate a class of self-destructing polymer capsules that we call "motherships". The capsules are designed to hold a cargo of smaller structures (e.g., liposomes or fluorescent colloidal particles) that are collectively called "babyships" because they can hold payloads such as drugs and biomolecules either in their interiors or on their surfaces. Self-destructive properties

Received: March 8, 2013

Revised: April 23, 2013 


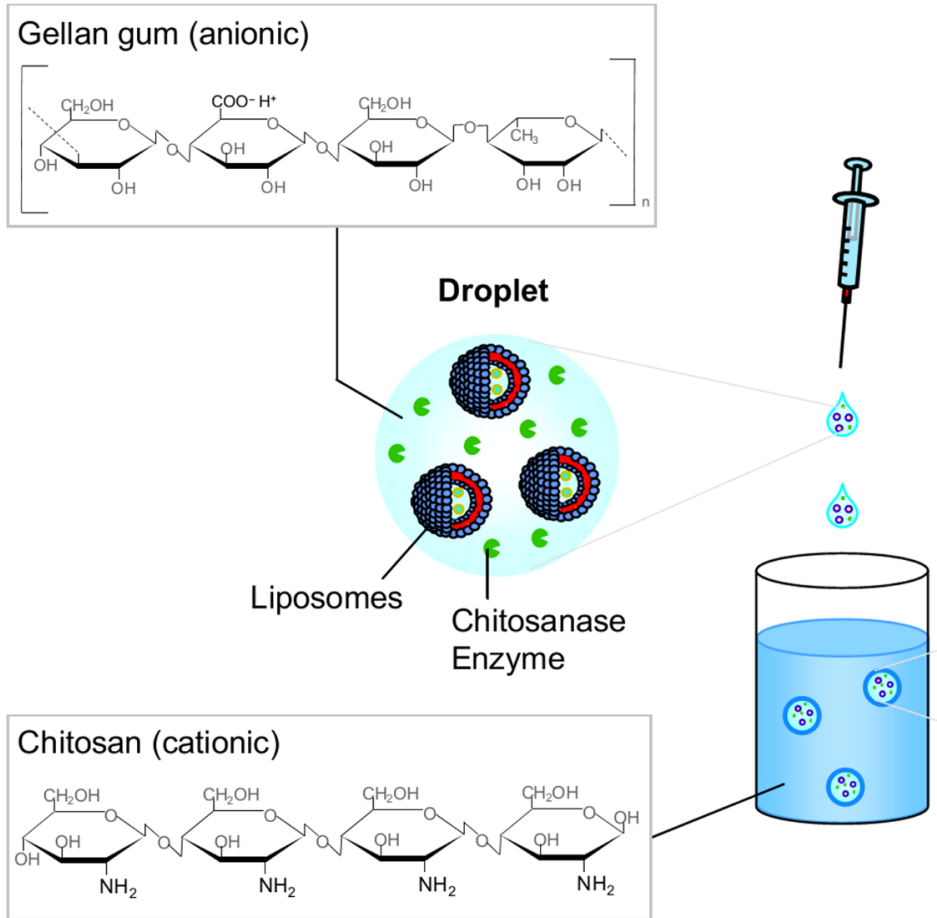

\section{Capsule}

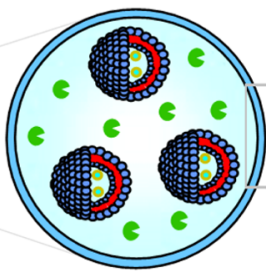

Interfacial Shell

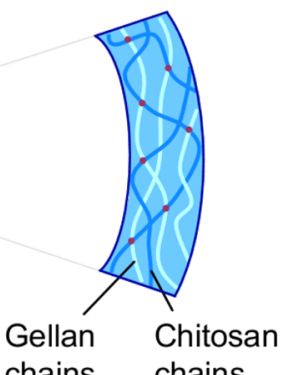

Figure 1. Preparation of self-destructing mothership capsules. The anionic polymer, gellan gum, is combined with liposomes and the enzyme, chitosanase, to form the feed solution. This is added dropwise to a solution of the cationic polymer, chitosan. In the process, the droplet is converted to a capsule with a rigid interfacial shell formed by electrostatic complexation of the oppositely charged polymers.

are imparted to the capsules by an additional element in their cargo, viz., enzymes that can break down the capsule shell. The capsules are made using two commercially available biopolymers, chitosan and gellan gum, as the precursors. ${ }^{13-16} \mathrm{We}$ show that mothership capsules can be created and loaded with the appropriate cargo in a single step, as illustrated in Figure 1. The enzyme used is chitosanase, which can hydrolyze chitosan and thus degrade the shell of these capsules. ${ }^{17,18}$ Mothership capsules can be stored as a dry powder, and when they are hydrated, their "internal clock" is initiated for the degradation of their shells. Upon degradation, the cargo of babyships is released en masse. The degradation time of motherships is shown to be tunable by the concentration of encapsulated enzyme and can be varied from minutes to hours.

\section{EXPERIMENTAL SECTION}

Polymers. Chitosan was obtained from Wako Pure Chemicals (product name Chitosan 1000). It is expected to have an average molecular weight of $1800000 \mathrm{Da} .{ }^{16}$ Because chitosan is soluble only under acidic conditions, $0.2 \mathrm{M}$ acetic acid was used to dissolve it. Gelrite gellan gum was obtained from Sigma-Aldrich, and its molecular weight was specified by the manufacturer to be 1000000 Da. Gellan gum solutions were prepared in deionized (DI) water by heating to 60 ${ }^{\circ} \mathrm{C}$.

Liposomes. The phospholipid L- $\alpha$-phosphatidylcholine (PC) was purchased from Avanti Polar Lipids Inc. Liposomes were prepared by an extrusion method, as recommended by the manufacturer. Briefly, dried films of the lipids were hydrated under moderate stirring. These were subjected to five freeze-thaw cycles and then passed through two double-stacked polycarbonate membrane filters (100 nm pores) using a Lipex pressurized extrusion system. This ensured liposomes of approximately $100 \mathrm{~nm}$ size, which was confirmed by dynamic light scattering. The fluorescent lipid $1,1^{\prime}$-dioctadecyl-3,3,3',3'-tetramethylindo-carbocyanine perchlorate (DiI) was obtained from SigmaAldrich. Liposomes stained with DiI were synthesized by mixing DiI and $\mathrm{PC}$ in a 1:9 molar ratio and preparing by the extrusion method mentioned above. This gave pink fluorescent vesicles that emit light at $567 \mathrm{~nm}$ when excited by light of wavelength $550 \mathrm{~nm}$.

Enzymes and Other Chemicals. Chitosanase from Streptomyces with an activity of 18 units/mg was obtained from Sigma-Aldrich and stored in a $50 \%$ glycerol solution containing $100 \mathrm{mM}$ sodium acetate at $\mathrm{pH}$ 5. (A unit is the amount of enzyme required to catalyze the conversion of one micromole of substrate per minute.) Carboxylatemodified polystyrene beads ( $2 \mu \mathrm{m}$, fluorescent yellow green) were also obtained from Sigma-Aldrich.

Dynamic Light Scattering (DLS). A Photocor-FC light-scattering instrument with a $5 \mathrm{~mW}$ laser light source at $633 \mathrm{~nm}$ was used at 25 ${ }^{\circ} \mathrm{C}$ with the scattering angle being $90^{\circ}$. The autocorrelation function was measured using a logarithmic correlator. Particle sizes were extracted from the autocorrelation function using the Dynals software package supplied by Photocor.

Fluorescence Measurements. A spectraMax M2e plate reader (Molecular Devices) was used to measure the fluorescence from DiIcontaining liposomes. The device uses the scan method of the SoftMax Pro software, measuring fluorescence at 21 different points on the plate and supplying the average fluorescence value on an internally calibrated scale.

Optical Microscopy. A Zeiss Axiovert 135 TV inverted microscope equipped with the Motic Image Plus imaging system was used for optical microscopy. Capsules were imaged with a $2.5 \times$ objective. Fluorescence microscopy of fluorescent microparticles was carried out with the excitation wavelength set at $480 \mathrm{~nm}$ and the emission filter at $510 \mathrm{~nm}$.

\section{RESULTS AND DISCUSSION}

We prepared self-destructing mothership capsules containing liposomes and enzyme by the procedure outlined in Figure 1. The method involves the electrostatic complexation of the cationic biopolymer, chitosan, and the anionic biopolymer, gellan gum. ${ }^{13-16}$ First, DiI-stained PC liposomes were prepared in DI water using the zwitterionic phospholipid PC. The liposomes are expected to be unilamellar structures, and their diameter was measured to be about $100 \mathrm{~nm}$ by DLS. The 
liposomes (0.5 wt \%) were then mixed with gellan gum (1 wt $\%)$ and the chitosanase enzyme ( 1.5 units $/ \mathrm{mL})$ to form the feeder solution. The feeder solution was loaded into a syringe and dispensed as droplets via the tip of a 22 gauge needle into a solution of chitosan (1 wt \%). The droplets were allowed to incubate for $3 \mathrm{~min}$, whereupon each droplet was converted to a capsule (i.e., a shell was formed on the surface of the droplet by the electrostatic complexation of chitosan and gellan chains into a polyion network). The resulting capsules are the motherships, and they contain the liposomes and enzyme inside. These capsules take up the pink color of the internalized stained liposomes. They were mechanically separated from the chitosan solution with a spatula and washed three times with DI water. The capsules were then stored in phosphate buffer $(\mathrm{pH} \mathrm{6})$ solution, where they remained stable under mechanical stirring. Note that the size of the capsules can be controlled by adjusting the size of the generated drop. For our purposes here, we mostly have prepared capsules with sizes $>1 \mathrm{~mm}$. Smaller capsules can be created by reducing the drop size using microfluidic or atomization techniques. ${ }^{19,20}$

The self-destructing property of the capsules could be characterized by optical microscopy as well as visual observation. Figure 2, panels $\mathrm{A}-\mathrm{C}$ are micrographs of a

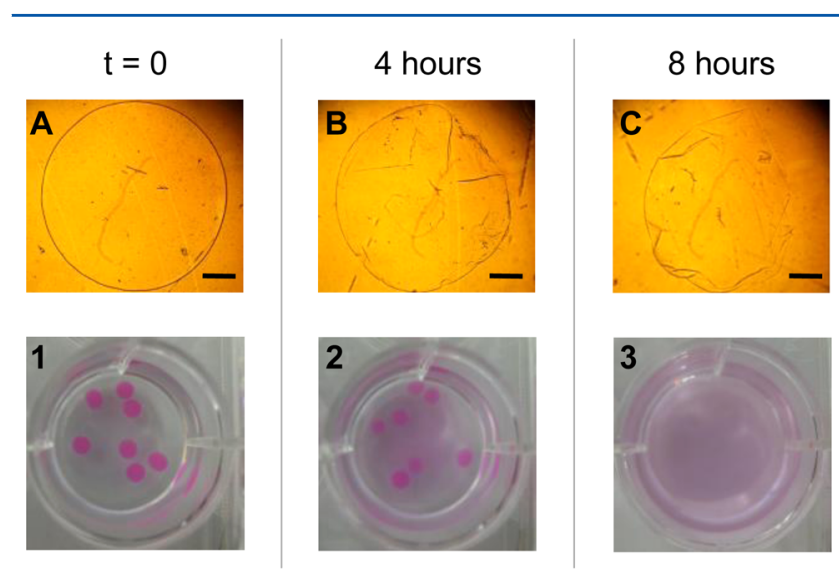

Figure 2. Optical micrographs and visual observations of mothership capsules undergoing self-destruction. The capsules contain nanoscale liposomes and 1.5 units $/ \mathrm{mL}$ of chitosanase enzyme in their lumen. (A-C) Optical micrographs showing the self-destruction of a capsule. The scale bar in these images is $500 \mu \mathrm{m}$. (A) Initially the capsule shell is intact. (B) After $4 \mathrm{~h}$, the shell is partially degraded. (C) After $8 \mathrm{~h}$, the shell is extensively degraded. (1-3) Visual observations of capsules in solution undergoing self-destruction. The liposomes are fluorescently tagged and impart a pink color to the capsules. (1) Initially, capsules are intact. (2) After $4 \mathrm{~h}$, the capsules are partially degraded, releasing liposomes, as evidenced by the appearance of a pink color in the solution. (3) The capsules are substantially degraded and no longer visible to the eye; in turn, the solution shows a uniform pink color.

mothership capsule as it undergoes degradation via internalized chitosanse enzyme. Initially, the capsule is intact and its shell is uncompromised. After $4 \mathrm{~h}$, the shell is seen to be partially degraded, but the capsule still largely retains its shape. After $8 \mathrm{~h}$, the shell is sufficiently degraded and ruptured such that the capsule is no longer able to retain its internal content. Control capsules with no encapsulated enzyme showed no degradation or loss of mechanical integrity within the same time frame. Thus, it is clear that the changes in the capsule in Figure 2 are due to the enzymatic action of chitosanase, which is known to cleave the $\beta$ - $(1,4)$ linkages between the D-glucosamine units in chitosan and thus degrade the polymeric chains into short oligomers. $^{17,18}$ Because the capsule shell is formed by the complexation of gellan and chitosan chains, the enzymatic degradation of the chitosan rutpures the shell and thereby destroys the capsule.

A key aspect of our self-destructing motherships is that the babyships (i.e., liposomes) are released intact into the surroundings. Evidence for such release in provided in Figure 2 , panels $1-3$. The liposomes in this study were of $\sim 100 \mathrm{~nm}$ diameter and were formulated with the fluorescent lipid DiI. This lipid incorporates itself into the liposomal bilayer; in the absence of a bilayer, DiI molecules on their own are not soluble in water. Intact liposomes display a pink color because of DiI in the bilayer, but if the liposomes are disrupted, then DiI will precipitate out in the external solution. Panel 1 shows a number of intact mothership capsules in a buffer solution at time zero. The capsules have a pink color because of the DiI-tagged liposomes in their lumen. Note that the pink color is confined entirely to the capsules. At the $4 \mathrm{~h}$ mark, the capsules have lost their integrity because of the enzymatic degradation of the shell, and in turn, there is some release of liposomes, as seen from the emergence of a pink color in the solution. After $8 \mathrm{~h}$, the capsules are substantially degraded, releasing most of their liposomes into the surrounding solution. The solution shows a nearly uniform pink color (no precipitate), and a DLS measurement on this solution gave an average diameter of $100 \mathrm{~nm}$, which matches that of the original liposomes. These observations provide indirect evidence that the motherships release intact liposomes.

Next, we studied the effect of chitosanase concentration on the degradation profile of gellan-chitosan mothership capsules. Because DiI is a fluorescent molecule, the release of DiI-stained liposomes as the capsules self-destruct causes an increase in fluorescence in the external buffer solution. We measured this fluorescence at regular time intervals, and Figure 3a shows the release profiles for mothership capsules containing two different amounts of enzyme. Note that the release is much more rapid at the higher enzyme concentration. Negligible fluorescence in the external solution was observed in the control case (i.e., capsules not containing enzyme) over $10 \mathrm{~h}$, indicating that liposomes cannot leak out of intact capsules. To quantify these release experiments, we fix the time to release half of the liposomes (i.e., for the cumulative release to reach 50\%) as a measure of the degradation time. Thus, from the curves in Figure $3 \mathrm{a}$, the degradation time is $2 \mathrm{~h}$ for 3.0 units $/ \mathrm{mL}$ of enzyme and $4.9 \mathrm{~h}$ for 1.5 units $/ \mathrm{mL}$ of enzyme. From a series of such experiments, we create a plot of degradation time versus enzyme concentration, which is shown in Figure $3 \mathrm{~b}$. The data reveal a monotonic decrease in degradation time with enzyme concentration until $\sim 3$ units $/ \mathrm{mL}$. At concentrations above this value, the degradation time becomes almost constant at $\sim 2 \mathrm{~h}$. Overall, by varying the concentration of internalized enzyme, we can access a 4-fold range in the degradation time. At this point, it may be worthwhile to add that we were also able to degrade mothership capsules by placing the chitosanase in the external buffer solution instead of inside the capsule.

In addition to the release of liposomes, we also demonstrate the release of a different payload from the mothership capsules. We encapsulated fluorescent polystyrene microparticles (diameter, $2 \mu \mathrm{m}$ ) instead of liposomes within capsules bearing 1.5 units/mL of enzyme. The self-destruction of these capsules in buffer was monitored by fluorescence microscopy. Figure $4 \mathrm{a}$ shows that at $t=0$ the particles are localized in the interior of 

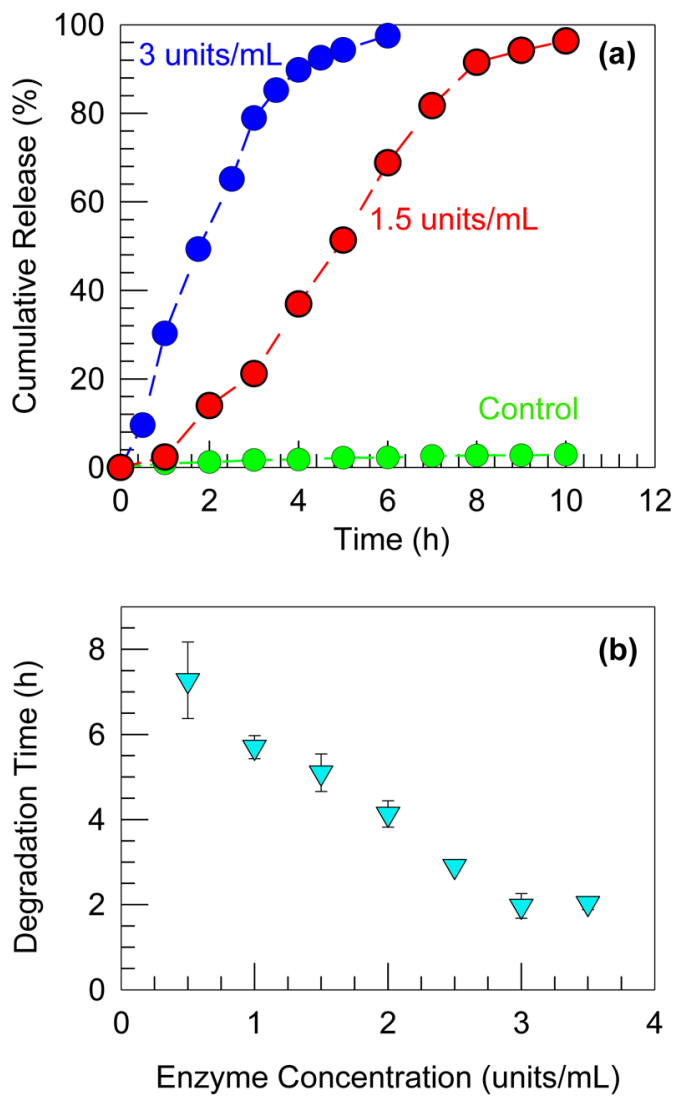

Figure 3. Quantifying the release of liposomes from self-destructing mothership capsules. (a) Liposomal release profiles as a function of time, as measured by the increase in fluorescence in solution, for two different enzyme concentrations. A faster release is seen for the higher concentration. The control sample contains no enzyme and shows no release of liposomes. (b) Degradation time (estimated as the time corresponding to $50 \%$ release) as a function of enzyme concentration. The degradation time decreases with increasing enzyme until it saturates at around 3 units $/ \mathrm{mL}$ of enzyme. Error bars for each point represent the standard deviation among three runs.

the capsule. A shell region with a thickness of a few micrometers surrounds the particle-filled lumen (see arrow). The fluorescence signal is confined to the capsules; no fluorescence is observed in the background. As the capsules self-destruct, some fluorescence (green color) is evident in the background at the $3 \mathrm{~h}$ mark (Figure $4 \mathrm{~b}$ ), indicating that the enzyme has partially degraded the capsule shell and allowed some particles to leak out (see arrow). As time progresses, the particles diffuse out radially and extend beyond the breached shell (Figure 4c,d). At the same time, an increasing fluorescence signal is observed in the background. Interestingly, even after nearly complete degradation of the capsule shell, the particles are largely localized in the vicinity of the original capsule structure (unlike the liposomes in Figure 2, which are able to diffuse throughout the sample volume). This is because the diffusion of the larger fluorescent particles is $\sim 20$ times slower than that of the liposomes based on the Stokes-Einstein equation.

From the above results, it is evident that our self-destructing mothership capsules start degrading as soon as the capsules are prepared and placed in solution. For use in various applications, it would be preferable if we could delay the "clock" for starting the enzymatic self-destruction sequence. Thus, we studied (a) $t=0$

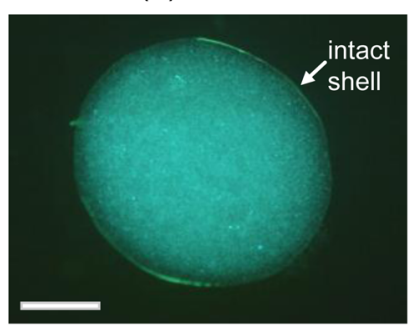

(c) 4 hours

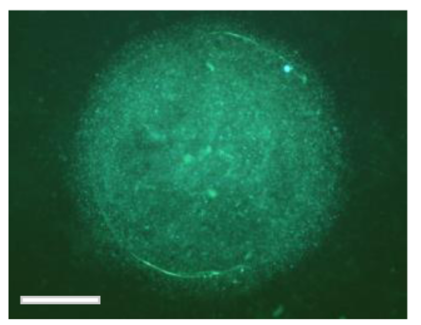

(b) 3 hours

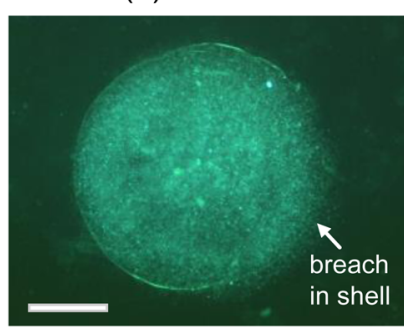

(d) 5 hours

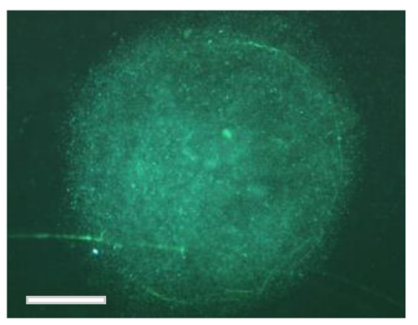

Figure 4. Release of fluorescent microparticles from self-destructing mothership capsules. Fluorescence micrographs are shown at various times for a capsule containing 1.5 units $/ \mathrm{mL}$ of chitosanase. (a) Initially, the particles are confined to the interior of the capsule. The outline of the capsule shell is clearly visible. (b) After $3 \mathrm{~h}$, the capsule is partially degraded and a breach is visible in the right corner of the shell, which allows some particles to escape. (c) After $4 \mathrm{~h}$ and (d) after $5 \mathrm{~h}$, there is further progressive degradation of the capsule and in turn the particles diffuse radially outside the confines of the shell. Correspondingly, the fluorescence in the background becomes appreciable relative to the initial case. The scale bar in each image is $1000 \mu \mathrm{m}$.

whether these capsules could be freeze-dried and stored and if they would then reactivate once rehydrated. Figure 5 shows that this is indeed the case. We took freshly prepared mothership capsules and immediately subjected them to freeze-drying under mild conditions. Dehydrated capsules collapsed to a dry powder (Figure 5a), allowing for convenient storage. Subsequently, the freeze-dried capsules were reconstituted by hydrating with a $\mathrm{pH} 6$ buffer solution. Hydration caused the capsules to regain their original form, as shown in Figure $5 b$. Additionally, capsules regained their self-destructing nature, undergoing degradation as expected and following the same kinetics as capsules that had not had their internal clock halted by freeze-drying (Figure 5c). Control capsules without enzyme could also be rehydrated after freeze-drying but did not undergo any degradation. Thus, by employing a simple freezedrying method, we have demonstrated that self-destructing motherships can be stored until needed for a particular application.

\section{CONCLUSIONS}

We have synthesized mothership capsules, which are polymeric containers that encapsulate much smaller babyships. The latter can be nanocontainers such as liposomes or colloidal particles. A simple, single-step method is used to generate these capsules under mild conditions starting from the biopolymers chitosan and gellan gum. Additionally, we have designed motherships that can self-destruct because of an encapsulated enzyme. The enzyme breaks down the polymeric shell of the capsule and thereby allows the liposomes to escape intact. The capsule degradation time can be tuned by the concentration of 
(a) Freeze-dried mothership capsules
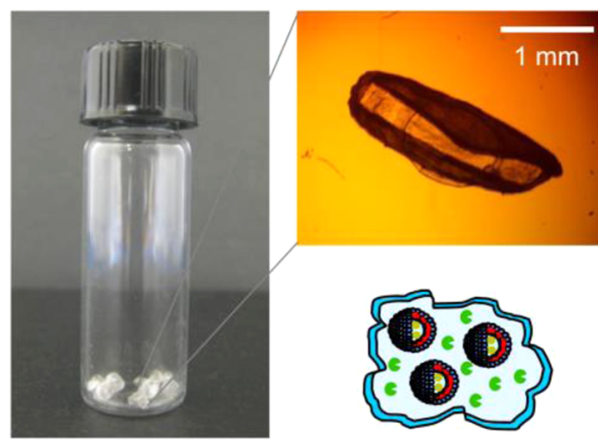

(b) Rehydrate with buffer
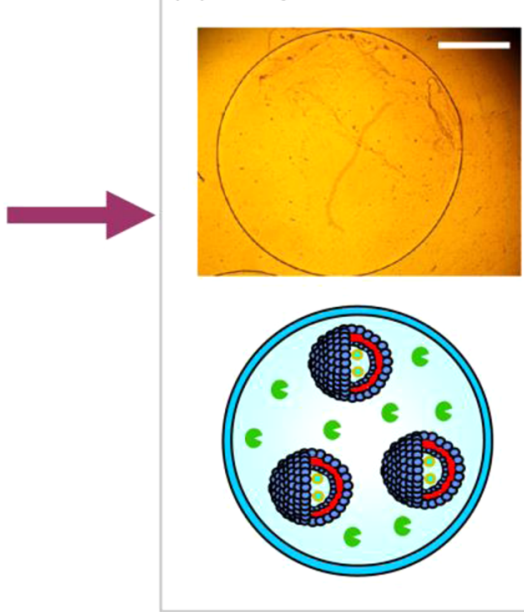

(c) Capsules self-destruct
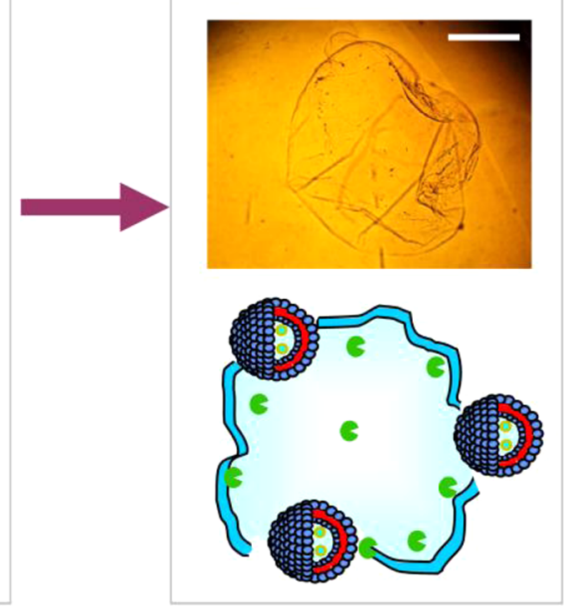

Figure 5. Demonstration that mothership capsules can be stored and subsequently reactivated. (a) Freshly prepared mothership capsules are freezedried and stored as a powder. An optical micrograph of a freeze-dried capsule is shown along with a schematic of this structure. (b) Micrograph and schematic of a capsule that has been reconstituted by placing in aqueous buffer. (c) Micrograph and schematic of a reconstituted capsule undergoing self-destruction. The scale bar in images $\mathrm{b}$ and $\mathrm{c}$ is $750 \mu \mathrm{m}$.

encapsulated enzyme. The motherships can be stored as an inert, freeze-dried powder until required; when reconstituted with buffer, their self-destruction sequence is activated. We believe that such hierarchical capsules have the potential for the delivery of a variety of payloads including drugs, biological therapeutics, cosmetics, and flavor ingredients. Different payloads can be encapsulated in the capsule lumen or within the liposomes. The concepts shown here can be easily extended to other polymer-enzyme pairs and also to structures over a range of sizes.

\section{AUTHOR INFORMATION}

\section{Corresponding Author}

*E-mail: sraghava@umd.edu.

\section{Author Contributions}

${ }^{\S}$ Equal contributions.

\section{Notes}

The authors declare no competing financial interest.

\section{ACKNOWLEDGMENTS}

This work was partially funded by grants from the NSF and TEDCO. M.B.D. was supported by a Fischell Fellowship from the Fischell Department of Bioengineering at UMD. We are grateful to Prof. Bill Bentley and Prof. John Fisher for allowing us the use of characterization tools in their laboratories. We also acknowledge helpful discusions with Prof. Greg Payne and Prof. Hamid Ghandehari.

\section{REFERENCES}

(1) De Geest, B. G.; De Koker, S.; Sukhorukov, G. B.; Kreft, O.; Parak, W. J.; Skirtach, A. G.; Demeester, J.; De Smedt, S. C.; Hennink, W. E. Polyelectrolyte microcapsules for biomedical applications. Soft Matter 2009, 5, 282-291.

(2) Esser-Kahn, A. P.; Odom, S. A.; Sottos, N. R.; White, S. R.; Moore, J. S. Triggered release from polymer capsules. Macromolecules 2011, 44, 5539-5553.

(3) Kibat, P. G.; Igari, Y.; Wheatley, M. A.; Eisen, H. N.; Langer, R. Enzymatically activated microencapsulated liposomes can provide pulsatile drug release. FASEB J. 1990, 4, 2533-2539.
(4) Machluf, M.; Regev, O.; Peled, Y.; Kost, J.; Cohen, S. Characterization of microencapsulated liposome systems for the controlled delivery of liposome-associated macromolecules. J. Controlled Release 1997, 43, 35-45.

(5) Lee, H. Y.; Tiwari, K. R.; Raghavan, S. R. Biopolymer capsules bearing polydiacetylenic vesicles as colorimetric sensors of $\mathrm{pH}$ and temperature. Soft Matter 2011, 7, 3273-3276.

(6) Stenekes, R. J. H.; Loebis, A. E.; Fernandes, C. M.; Crommelin, D. J. A.; Hennink, W. E. Degradable dextran microspheres for the controlled release of liposomes. Int. J. Pharm. 2001, 214, 17-20.

(7) Itoh, Y.; Matsusaki, M.; Kida, T.; Akashi, M. Enzyme-responsive release of encapsulated proteins from biodegradable hollow capsules. Biomacromolecules 2006, 7, 2715-2718.

(8) Itoh, Y.; Matsusaki, M.; Kida, T.; Akashi, M. Time-modulated release of multiple proteins from enzyme-responsive multilayered capsules. Chem. Lett. 2008, 37, 238-239.

(9) Broaders, K. E.; Pastine, S. J.; Grandhe, S.; Frechet, J. M. J. Aciddegradable solid-walled microcapsules for $\mathrm{pH}$-responsive burst-release drug delivery. Chem. Commun. 2011, 47, 665-667.

(10) De Geest, B. G.; Dejugnat, C.; Sukhorukov, G. B.; Braeckmans, K.; De Smedt, S. C.; Demeester, J. Self-rupturing microcapsules. Adv. Mater. 2005, 17, 2357-2361.

(11) De Geest, B. G.; Dejugnat, C.; Prevot, M.; Sukhorukov, G. B.; Demeester, J.; De Smedt, S. C. Self-rupturing and hollow microcapsules prepared from bio-polyelectrolyte-coated microgels. Adv. Funct. Mater. 2007, 17, 531-537.

(12) Borodina, T.; Markvicheva, E.; Kunizhev, S.; Moehwald, H.; Sukhorukov, G. B.; Kreft, O. Controlled release of DNA from selfdegrading microcapsules. Macromol. Rapid Commun. 2007, 28, 18941899.

(13) Amaike, M.; Senoo, Y.; Yamamoto, H. Sphere, honeycomb, regularly spaced droplet and fiber structures of polyion complexes of chitosan and gellan. Macromol. Rapid Commun. 1998, 19, 287-289.

(14) Yamamoto, H.; Senoo, Y. Polyion complex fiber and capsule formed by self-assembly of chitosan and gellan at solution interfaces. Macromol. Chem. Phys. 2000, 201, 84-92.

(15) Ohkawa, K.; Kitagawa, T.; Yamamoto, H. Preparation and characterization of chitosan-gellan hybrid capsules formed by selfassembly at an aqueous solution interface. Macromol. Mater. Eng. 2004, 289, 33-40.

(16) Fujii, T.; Ogiwara, D.; Ohkawa, K.; Yamamoto, H. Alkaline phosphatase encapsulated in gellan-chitosan hybrid capsules. Macromol. Biosci. 2005, 5, 394-400. 
(17) Boucher, I.; Dupuy, A.; Vidal, P.; Neugebauer, W. A.; Brzezinski, R. Purification and characterization of a chitosanase from Streptomyces N174. Appl. Microbiol. Biotechnol. 1992, 38, 188-193.

(18) Somashekar, D.; Joseph, R. Chitosanases - properties and applications: a review. Bioresour. Technol. 1996, 55, 35-45.

(19) Jiang, K. Q.; Xue, C.; Arya, C. D.; Shao, C. R.; George, E. O.; DeVoe, D. L.; Raghavan, S. R. A new approach to in-situ "micromanufacturing": microfluidic fabrication of magnetic and fluorescent chains using chitosan microparticles as building blocks. Small 2011, 7, 2470-2476.

(20) Kontturi, L. S.; Yliperttula, M.; Toivanen, P.; Maatta, A.; Maatta, A. M.; Urtti, A. A laboratory-scale device for the straightforward production of uniform, small sized cell microcapsules with long-term cell viability. J. Controlled Release 2011, 152, 376-381. 